\author{
Jean-Michel Liet \\ Jean-Marc Dejode \\ Nicolas Joram \\ Bénédicte Gaillard Le Roux \\ Yann Péréon
}

\section{Bedside diagnosis of bilateral diaphragmatic paralysis}

Accepted: 1 October 2012

Published online: 31 October 2012

(C) Springer-Verlag Berlin Heidelberg and ESICM 2012

\section{Dear Editor,}

Bilateral diaphragmatic paralysis

(BDP) is a rare cause of respiratory failure that is quite challenging to diagnose [1]. Here we describe the cases of two children in whom BDP was easily detected at bedside once we used an esophagal probe equipped with sensors that measured the electrical activity of the diaphragm.

A 3-year-old boy with complex congenital cardiopathy underwent a third surgery using the bidirectional Glenn anastomosis procedure. After extubation, he developed intense dyspnea. A commercially available feeding tube equipped with sensors (Edi 8-Fr catheter, Maquet Critical Care, Solna, Sweden) was installed to provide respiratory support using the neurally adjusted ventilatory assist mode (NAVA). This esophageal probe records electrical activity of the diaphragm via a Servo-I Ventilator (Maquet Critical Care, Solna, Sweden) using a standardized method [2]. The child regained spontaneous respiratory activity, but no electrical activity of the diaphragm could be found, thus evoking the diagnosis of BDP. This diagnosis was confirmed by a fluoroscopy. The child was completely liberated from noninvasive ventilation on the 18 th postoperative day.

A 9-month-old girl with an atrioventricular canal defect underwent a third surgery for mitral valve replacement. Over the next few days, she experienced two failed extubations. Echocardiographies showed good cardiac function. Laryngotracheal fibroscopy did not provide an explanatory diagnosis. A fluoroscopy of the diaphragm receiving pressure support ventilation was carried out by a radiologist who concluded that there were no components indicating diaphragmatic paralysis. A thoracic tomodensitometry showed systematized alveolar condensations reminiscent of non-specific ventilatory disorders. Two weeks after the valve replacement, a pacemaker was implanted because of a persistent complete atrioventricular block. Thereafter, the attending physician decided to try ventilatory support using the NAVA mode because of the good results we had obtained in other cases of challenging ventilation [3]. The respiratory rate and ventilator pressures were reduced in order to restore spontaneous respiratory activity. Nevertheless, the Edi catheter did not detect any electrical activity of the diaphragm. The diagnosis of BDP was then confirmed by an electromyography of the diaphragm with a phrenic nerve conduction study. After a left diaphragmatic plication, the child was free of all mechanical ventilation 3 weeks later.

Bilateral diaphragmatic paralysis is a rare cause of respiratory distress that develops in various neuropathies and myopathies [1]. After cardiac surgery BDP is typically due to traction applied during the thoracic surgery [4]. Bilateral diaphragmatic paralysis is difficult to diagnose as there are no asymmetric movements, unlike unilateral diaphragmatic paralysis. Fluoroscopic imaging, although sensitive and specific for unilateral diaphragm paralysis, is frequently misinterpreted when diaphragmatic paralysis is bilateral.

Currently, measurement of electrical activity of the diaphragm can be done at the bedside with a specific esophageal probe connected to a commercially available ventilator [5]. The position of this probe must be carefully checked using the positioning window and ECG signals. The absence of electrical activity of the diaphragm in a child with unexplained respiratory distress, where spontaneous breathing activity is present, strongly suggests the diagnosis of BDP.

Conflicts of interest None.

\section{References}

1. Billings ME, Aitken ML, Benditt JO (2008) Bilateral diaphragm paralysis: a challenging diagnosis. Respir Care 53:1368-1371

2. Sinderby C, Navalesi P, Beck J, Skrobik Y, Comtois N, Friberg S et al (1999) Neural control of mechanical ventilation in respiratory failure. Nat Med 5:1433-1436. doi:10.1038/71012

3. Liet JM, Dejode JM, Joram N, GaillardLe Roux B, Betremieux P, Roze JC (2011) Respiratory support by neurally adjusted ventilatory assist (NAVA) in severe RSV-related bronchiolitis: a case series report. BMC Pediatr 11:92. doi: 10.1186/1471-2431-11-92

4. Dagan O, Nimri R, Katz Y, Birk E, Vidne B (2006) Bilateral diaphragm paralysis following cardiac surgery in children: 10-years' experience. Intensive Care Med 32:1222-1226. doi: 10.1007/s00134-006-0207-5

5. Bordessoule A, Emeriaud G, Delnard N, Beck J, Jouvet P (2010) Recording diaphragm activity by an oesophageal probe: a new tool to evaluate the recovery of diaphragmatic paralysis. Intensive Care Med 36:1978-1979. doi: 10.1007/s00134-010-1963-9

J.-M. Liet $(\bowtie)$ · J.-M. Dejode · N. Joram B. Gaillard Le Roux CHU de Nantes, Pediatric Critical Care Unit, Hopital Mere-Enfant, Nantes, France e-mail: jeanmichel.liet@chu-nantes.fr

Y. Péréon

CHU de Nantes, Laboratoire d'explorations fonctionnelles, Hotel Dieu, Nantes, France 